Published in final edited form as:

Birth. 2013 December ; 40(4): 221-226. doi:10.1111/birt.12062.

\title{
Baby-Friendly Hospital Practices and Birth Costs
}

\author{
Jessica A. Allen, MPH, MSW, \\ Division of Nutrition, Physical Activity, and Obesity, Centers for Disease Control and Prevention \\ Holly B. Longenecker, MSPH, \\ Northrop Grumman Corporation, Atlanta, GA, USA \\ Cria G. Perrine, PhD, and \\ Division of Nutrition, Physical Activity, and Obesity, Centers for Disease Control and Prevention \\ Kelley S. Scanlon, PhD, RD \\ Division of Nutrition, Physical Activity, and Obesity, Centers for Disease Control and Prevention
}

\section{Abstract}

Background-Hospital practices supportive of breastfeeding can improve breastfeeding rates. There are limited data available on how improved hospital practices are associated with hospital costs. We describe the association between the number of breastfeeding supportive practices a hospital has in place and the cost of an uncomplicated birth.

Methods-Data from hospitals in 20 states that participated in the 2007 Maternity Practices in Infant Nutrition and Care (mPINC) survey and Healthcare Cost and Utilization Project's (HCUP) State Inpatient Databases (SID) were merged to calculate the average median hospital cost of uncomplicated vaginal and cesarean section births by number of ideal practices from the Ten Steps to Successful Breastfeeding. Linear regression analyses were conducted to estimate change in birth cost for each additional ideal practice in place.

Results-Sixty-one percent of hospitals had ideal practice on 3-5 of the 10 steps, whereas 29 percent of hospitals had ideal practice on 6-8. Adjusted analyses of uncomplicated births revealed a higher but nonsignificant increase in any of the birth categories (all births, $\$ 19$; vaginal, $\$ 15$; cesarean section, \$39) with each additional breastfeeding supportive maternity care practice in place.

Conclusions-Our results revealed that the number of breastfeeding supportive practices a hospital has in place is not significantly associated with higher birth costs. Concern for higher birth costs should not be a barrier for improving maternity care practices that support women who choose to breastfeed.

\footnotetext{
Address correspondence to: Jessica A. Allen, MPH, MSW, Centers for Disease Control and Prevention, 4770 Buford Hwy NE, Mailstop F-77, Atlanta, GA 30341, USA.

The authors have no financial relationships to disclose and no conflicts of interest.

The findings and conclusions in this report are those of the authors and do not necessarily represent the official position of the Centers for Disease Control and Prevention.
} 


\section{Keywords}

breastfeeding; maternity care practices; birth costs

The health benefits of breastfeeding are well established. Infants who are breastfed experience fewer episodes of diarrhea, ear infections, and lower respiratory tract infections and are at a reduced risk of Sudden Infant Death Syndrome, diabetes, and obesity $(1,2)$. Mothers who breastfeed have a reduced risk of breast and ovarian cancer (1). These health benefits contribute to a reduction in the direct and indirect medical costs associated with breastfeeding (3).

Although experts recommend that infants should be exclusively breastfed for about 6 months, at 7 days after birth approximately half of infants in the United States are receiving infant formula $(4,5)$. Hospital practices that a woman experiences during her maternity stay affect her ability to start and continue breastfeeding and to meet her own exclusive breastfeeding intentions (6-10). In 1991, the World Health Organization (WHO) and United Nations Children's Fund (UNICEF) developed the Baby-Friendly Hospital Initiative, the core of which is implementation of the Ten Steps to Successful Breastfeeding, outlining 10 evidence-based steps that hospitals can implement to support breastfeeding (Table 1) (11). In 2009, the American Academy of Pediatrics endorsed the Ten Steps to Successful Breastfeeding for the United States (12). To be designated as "Baby-Friendly" in the United States, a hospital must implement the Ten Steps to Successful Breastfeeding, follow the International Code of Marketing of Breastmilk Substitutes, and pass an on-site assessment conducted by Baby-Friendly Hospital Initiative, United States (13).

There are limited data available on whether implementation of the Ten Steps to Successful Breastfeeding affects hospital birth costs. Birth costs could be higher at hospitals implementing maternity care practices to support breastfeeding because facilities need to provide training on lactation for staff and pay for formula (14). In addition, the process of conducting quality improvement requires staff resources, which may only be feasible in facilities that have higher charges. On the other hand, some maternity care practices that support breastfeeding may reduce staff demands. For example, with 24-hour rooming-in there exists no need for staff to feed and care for infants in a newborn nursery. A 2010 study by DelliFraine et al compared the cost of a birth at Baby-Friendly designated hospitals with non Baby-Friendly designated hospitals in the United States and found the cost of a birth slightly, but not significantly, higher at Baby-Friendly designated hospitals (15). Many states have chosen to emphasize incremental implementation of all or some of the Ten Steps to Successful Breastfeeding to improve maternity practices, rather than immediately pursuing Baby-Friendly designation for their hospitals. Examples include the Colorado Can Do 5!, North Carolina's Five Star Breastfeeding-Friendly Designation, and Texas Ten Step Program (16-18). Given these initiatives, it is important to understand how incremental improvements in maternity care practices may affect hospital birth costs.

Our objective was to build on prior research by exploring the cost of a birth by the number of breastfeeding supportive practices in place at a hospital, using 10 indicators consistent with the WHO/UNICEF Ten Steps to Successful Breastfeeding: 1) existence of a model 
breastfeeding policy, 2) staff competency assessment, 3) prenatal breastfeeding education, 4) early initiation of breastfeeding, 5) teaching breastfeeding techniques, 6) limited supplementation of breastfeeding infants, 7) rooming-in, 8) teaching feeding cues, 9) limited use of pacifiers, and 10) post discharge support (19).

\section{Methods}

\section{Sample}

We used data from two sources for this analysis. The 2007 Maternity Practices in Infant Nutrition and Care survey (mPINC) is a national census of hospital maternity care practices conducted every 2 years by the Centers for Disease Control and Prevention. The survey is sent to all facilities with registered maternity beds in the United States and measures infant feeding related policies and practices and is completed by the person most knowledgeable about the relevant practices. mPINC contains 10 indicators of ideal practice consistent with WHO/UNICEF's Ten Steps to Successful Breastfeeding (20). The specific definitions used to describe ideal practice for each of the mPINC indicators have been described previously (19).

The second data source is the 2007 Healthcare Cost and Utilization Project's (HCUP) State Inpatient Databases (SID). These data are collected by the Agency for Healthcare Research and Quality (AHRQ) (21). SID contains inpatient discharge abstracts from primarily community hospitals, which are defined by the American Hospital Association (AHA) as all nonfederal, short-term, general, and other specialty hospitals (22). Twenty states had SID data available through AHRQ that included both AHA identification (AHA ID) and cost-tocharge ratio information. AHA ID was required to link SID to mPINC. The 20 states included were Arkansas, Arizona, California, Colorado, Florida, Iowa, Kentucky, Massachusetts, Maryland, North Carolina, New Jersey, Nevada, New York, Oregon, Rhode Island, Utah, Vermont, Washington, Wisconsin, and West Virginia.

Using diagnosis-related groups (DRG) we identified charges associated with uncomplicated vaginal (DRG 373) and uncomplicated cesarean section (DRG 371) singleton births. Charge information available on SID files is the amount a hospital bills for services. To estimate hospital costs we applied HCUP All-Payer Inpatient Cost-to-Charge (APICC) ratio to charge data. APICC is a hospital-specific, cost-to-charge ratio developed from hospital accounting reports collected by the Centers for Medicare and Medicaid Services. This ratio allows hospital charges to be translated into estimated costs to the facility (23).

After linking mPINC and SID files for 1052 hospitals in 20 states with SID data and excluding hospitals without complete information for cost (174 facilities) or for all 10 ideal hospital practices (164 facilities) our final sample included 747 hospitals. Hospitals included in our analysis did not differ in terms of cesarean section rates, annual number of births, or region compared with those excluded. They did differ on percent of Medicaid births and type of facility; compared with hospitals in the final analytic sample, hospitals excluded from the sample had a lower percent of Medicaid births (48\% vs 43\%), a higher percent were government ( $17 \%$ vs $19 \%)$, private ( $10 \%$ vs $16 \%)$, and military facilities ( $0 \%$ vs $5 \%$ ) and a lower percent were nonprofit facilities ( $73 \%$ vs $60 \%)$. 


\section{Statistical Analyses}

The unit of analysis was median birth cost at each hospital for total uncomplicated births, uncomplicated vaginal births, and uncomplicated cesarean section births. Median rather than average birth costs for each hospital were used because average birth costs were not normally distributed. We calculated the average of hospital-level median birth costs by number of ideal practices reported on mPINC. In addition, we used linear regression with hospital-level median birth cost as the outcome and number of ideal practices as the main predictor to estimate the change in birth cost associated with each additional ideal practice in place (from 0 to 10 practices). Final models were assessed for appropriateness to meet model assumptions for linear regression. We ran bivariate analyses, and models adjusting for percent of uncomplicated births covered by Medicaid, state, facility type (government, nonprofit, private, and military) and facility annual number of births $(<250,250-499,500$ 999, 1000-1999, 2000-4999, > 4999). We also compared average median birth costs between hospitals reporting $0-2$ practices with hospitals that provided 9-10 practices using a Satterthwaite $t$ test. All analyses were conducted in SAS 9.2 and $p$-values $<0.05$ were considered significant.

\section{Results}

Nearly three-quarters of the hospitals in the sample were nonprofit with the remaining facilities being either government or private. Sixty-one percent of facilities were implementing 3-5 ideal practices, with another 29 percent implementing 6-8 ideal practices. The mean number of births per year was 1488 and 43 percent of births were covered by Medicaid (Table 2). The group with the highest median birth cost for all uncomplicated births was hospitals reporting 9-10 ideal practices (\$3353) (Fig. 1); this finding was also true for vaginal (\$2971) and cesarean section (\$5722) births (Figs 2 and 3). However, the average birth cost at hospitals reporting $0-2$ practices was not significantly different from hospitals reporting 9-10 practices, regardless of birth mode $(p=0.27-0.49)$.

Unadjusted linear regression analyses of uncomplicated births revealed a significant increase in the cost of cesarean section births (\$98) with each additional practice in place, but no significant increase in the cost of all births or vaginal births ( $\$ 44$ and $\$ 35$, respectively) (Table 3). After adjustment for state, percent of births paid for by Medicaid, hospital type, and annual number of births, costs associated with additional practices were reduced compared with the unadjusted model, and were nonsignificant for all birth groups (all births, \$19; vaginal, \$15; cesarean section, \$39).

\section{Discussion}

Concerns that improving maternity care practices may lead to higher birth costs are understandable as combined charges for birthing women and newborns totaled over $\$ 79$ billion in 2005, and far exceeded total charges for any other condition (24). However, concerns that additional maternity care practices supportive of breastfeeding are associated with higher birth costs are not supported by our findings based on hospital records in 20 states, as we found no significant association between number of ideal practices reported and birth cost in the adjusted models. These findings are consistent with the findings of 
DelliFraine et al. Using two different methods to compare Baby-Friendly designated hospitals to non-Baby-Friendly hospitals their study found that costs were 1.6-5.0\% (\$35\$178) higher for Baby-Friendly hospitals, which was not a statistically significant difference (15). Similarly, we found a slightly higher but nonsignificant difference in birth costs associated with improved maternity care practices. Because non-Baby-Friendly hospitals may also have implemented some or even all of the Ten Steps to Successful Breastfeeding to improve maternity care practices, this study provides further evidence that improving hospital practices to support breastfeeding is not associated with significantly higher birth costs.

Our analysis is the first, to our knowledge, to examine the association between the incremental increase in number of ideal practices in place at a hospital and birth costs. In 2009, approximately half of all U.S. hospitals had 3-5 Baby-Friendly practices in place (19). Our data suggest that for these hospitals the cost of an uncomplicated birth would increase by approximately $\$ 95-\$ 133$ if they achieved all 10 practices; this analysis suggests the cost for uncomplicated vaginal and cesarean section births are not significantly higher for each additional ideal practice in place. Inherent in the process to become a Baby-Friendly hospital are expenses associated with designation fees, training, and purchasing infant formula (14). Neither the analysis by DelliFraine et al, nor our analysis was able to account for the costs associated with Baby-Friendly designation, or with implementing any particular practice. In fact very little data are available with respect to the cost of implementing the Ten Steps to Successful Breastfeeding and becoming Baby-Friendly. An article by Merewood and Philipp describes the experience of Boston Medical Center in becoming Baby-Friendly, including the cost of paying fair market value for infant formula (14). However, this article is now more than 10 years old, and is the experience of only one facility. Additional analyses, including longitudinal studies of change in cost as practices are implemented, are needed to better describe the costs associated with improving maternity care practices. In the meantime, this analysis suggests that whatever costs are assumed by a hospital in improving maternity care practices do not translate into increased birth costs at the facility.

Hospitals that follow the Ten Steps to Successful Breastfeeding increase breastfeeding initiation and in-hospital-exclusivity rates and improve patient care and quality $(25,26)$. In April 2010, the Joint Commission, an organization that accredits and certifies health care organizations in the United States, added the rate of in-hospital exclusive breastmilk feeding as a perinatal care core measure that can be tracked as part of quality improvement (27); this measure is in-line with step 6 , which indicates that breastfeeding infants should receive no food or drink other than breastmilk, unless medically indicated. Improving quality measures related to breastfeeding will help prepare hospitals seeking Baby-Friendly Hospital status, a designation a growing number of hospitals are obtaining (28).

This study has limitations. Birth costs were determined using overall, hospital-specific costto-charge ratios that only estimate birth costs and do not reflect the cost-to-charge ratio of specific units, such as labor and delivery. Besides, our sample of hospitals in 20 U.S. states is not nationally representative. Although the community hospitals included in this analysis included all uncomplicated births at the facility, hospitals without information for cost or ideal practices were excluded from analyses. However, the hospitals excluded did not differ 
from those included on important characteristics. Finally, mPINC is a self-reported survey filled out by a key informant at each hospital. Although a standard protocol is followed to identify the key informant, the responses may not accurately represent all practices.

In 2011, less than 50 percent of U.S. hospitals reported having more than five ideal practices in place (29). Hospitals may be helped in the adoption of these practices by better understanding the benefits and the barriers to adoption and how to overcome them.

\section{Conclusion}

This study did not find a significant increase in birth cost for each additional practice supportive of breast-feeding in place at a hospital. Breastfeeding is important for mothers, infants, and society and it is important that hospitals work to implement and improve maternity care practices that support breastfeeding and women who choose to breastfeed.

\section{References}

1. Ip S, Chung M, Raman G, et al. Breastfeeding and maternal and infant health outcomes in developed countries. Evid Rep Technol Assess (Full Rep). 2007 Apr.(153):1-186. Epub 2007/09/04. eng.

2. Owen C, Martin R, Whincup P, et al. Effect of infant feeding on the risk of obesity across the life course: a quantitative review of published evidence. Pediatrics. 2005; 115(5):1367-1377. [PubMed: 15867049]

3. Bartick M, Reinhold A. The Burden of Suboptimal Breastfeeding in the United States: A Pediatric Cost Analysis. Pediatrics. 2010; 125(5):e1048-e1056. [PubMed: 20368314]

4. American Academy of Pediatrics. Breastfeeding and the use of human milk. Pediatrics. 2012; 129(3):e827-e841. [PubMed: 22371471]

5. Centers for Disease Control and Prevention. [Accessed May 31, 2013] Breastfeeding among U.S. children born 2000-2009. CDC National Immunization Survey. 2012. Available at: http:// www.cdc.gov/breastfeeding/data/NIS_data/index.htm

6. Perrine C, Scanlon K, Li R, Odom E, Grummer SL. Baby-Friendly hospital practices and meeting exclusive breastfeeding intention. Pediatrics. 2012; 130(1):54-60. [PubMed: 22665406]

7. DiGirolamo A, Grummer SL, Fein S. Effect of maternity-care practices on breastfeeding. Pediatrics. 2008; 122(Suppl 2):S43-S49. [PubMed: 18829830]

8. Kramer MS, Chalmers B, Hodnett ED, et al. Promotion of Breastfeeding Intervention Trial (PROBIT): a randomized trial in the Republic of Belarus. JAMA. 2001; 285(4):413-420. [PubMed: 11242425]

9. Blomquist HK, Jonsbo F, Serenius F, Persson LA. Supplementary feeding in the maternity ward shortens the duration of breast feeding. Acta Paediatr. 1994; 83(11):1122-1126. [PubMed: 7841722]

10. World Health Organization. [Accessed October 30, 2012] Baby-friendly Hospital Initiative. Available at: http://www.who.int/nutrition/topics/bfhi/en/

11. World Health Organization. Evidence for the Ten Steps to Successful Breastfeeding. Geneva, Switzerland: World Health Organization; 1998.

12. American Academcy of Pediatrics. Author, 2009. Aug 25. 2009 Letter endorsing WHO/UNICEF Ten Steps to Successful Breastfeeding.

13. Baby-Friendly USA. [Accessed May 2013] Baby-Friendly USA: the gold standard of care. Available at: http://www.baby-friendlyusa.org/

14. Merewood A, Philipp BL. Implementing change: becoming baby-friendly in an inner city hospital. Birth. 2001; 28(1):36-40. [PubMed: 11264627]

15. DelliFraine J, Langabeer J, Williams JF, et al. Cost comparison of baby friendly and non-baby friendly hospitals in the United States. Pediatrics. 2011; 127(4):e989-e994. [PubMed: 21422086] 
16. Texas Ten Step. [Accessed May 31, 2013] Texas Ten Step Star Achiever Program. Available at: http://texastenstep.org/star-achiever-texastenstep/

17. Colorado Breastfeeding Coalition. [Accessed May 31, 2013] Colorado Can Do 5!. Available at: http://www.cobfc.org/co-can-do-5

18. North Carolina Nutrition Services Branch. [Accessed May 31, 2013] Five-Star BreastfeedingFriendly Designation. Available at: http://www.nutritionnc.com/breastfeeding/awardees.htm

19. Centers for Disease Control and Prevention (CDC). . Vital signs: hospital practices to support breastfeeding-United States, 2007 and 2009. Morb Mortal Wkly Rep. 2011; 60(30):1020-1025.

20. Centers for Disease Control and Prevention. [Accessed May 31, 2013] Maternity Practices in Infant Nutrition and Care. Available at: http://www.cdc.gov/breastfeeding/data/mpinc/index.htm

21. HCUP State Inpatient Databases (SID). Healthcare Cost and Utilization Project (HCUP). Rockville, Maryland: Agency for Healthcare Research and Quality; 2007.

22. American Hospital Association. [Accessed May 31, 2013] Fast Facts on US Hospitals. Available at: http://www.aha.org/research/rc/stat-studies/101207fastfacts.pdf

23. State Inpatient Database (SID) Healthcare Cost and Utilization Project (HCUP). [Accessed October 31, 2012] Cost-to-Charge Ratio Files: 2007 Central Distributor State Inpatient Database (CD-SID) User Guide. Available at: http://www.hcup-us.ahrq.gov/db/state/ CCR2007CDUserGuide.pdf

24. Sakala, C.; Corry, MP. Childbirth Connection, the Reforming States Group, and the Milbank Memorial Fund, Milbank Memorial Fund. 2008. Evidence-based maternity care: What it is and what it can achieve.

25. Declercq, ER.; Sakala, C.; Corry, MO.; Applebaum, S. Listening to Mothers II: Report of the Second National US Survey of Women's Childbearing Experiences. New York: Childbirth Connection; 2006.

26. Philipp BL, Merewood A, Miller LW, et al. Baby-friendly hospital initiative improves breastfeeding initiation rates in a US hospital setting. Pediatrics. 2001; 108(3):677-681. [PubMed: 11533335]

27. Joint Commission. [Accessed May 31, 2013] Specifications Manual for Joint Commission National Quality Measures (v2011A): Perinatal Care. Available at: http:// manual.jointcommission.org/releases/TJC2011A/PerinatalCare.html

28. Baby-Friendly USA. [Accessed. May 31, 2013] US Baby-Friendly Hospitals and Birth Centers. Available at: http://www.baby-friendlyusa.org/eng/index.html

29. Centers for Disease Control and Prevention. [Accessed September 23, 2013] Maternity Practices in Infant Nutrition and Care: mPINC Results Table 2011. Available at: http://www.cdc.gov/ breastfeeding/data/mpinc/data/2011/tables8_1a-8_2a.htm 


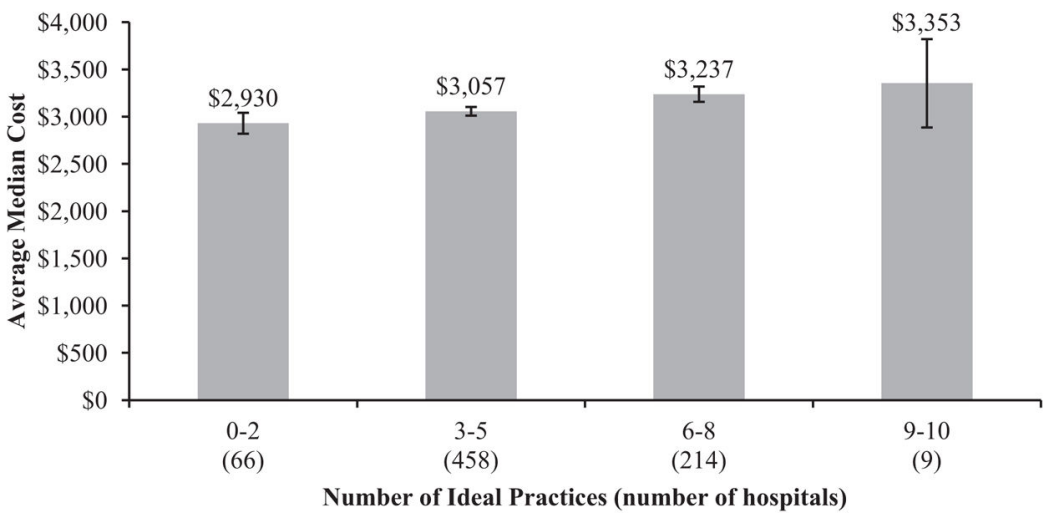

Figure 1.

Average median cost for all uncomplicated births by number of ideal practices. *Ideal practices are indicators of the Ten Steps to Successful Breastfeeding. 


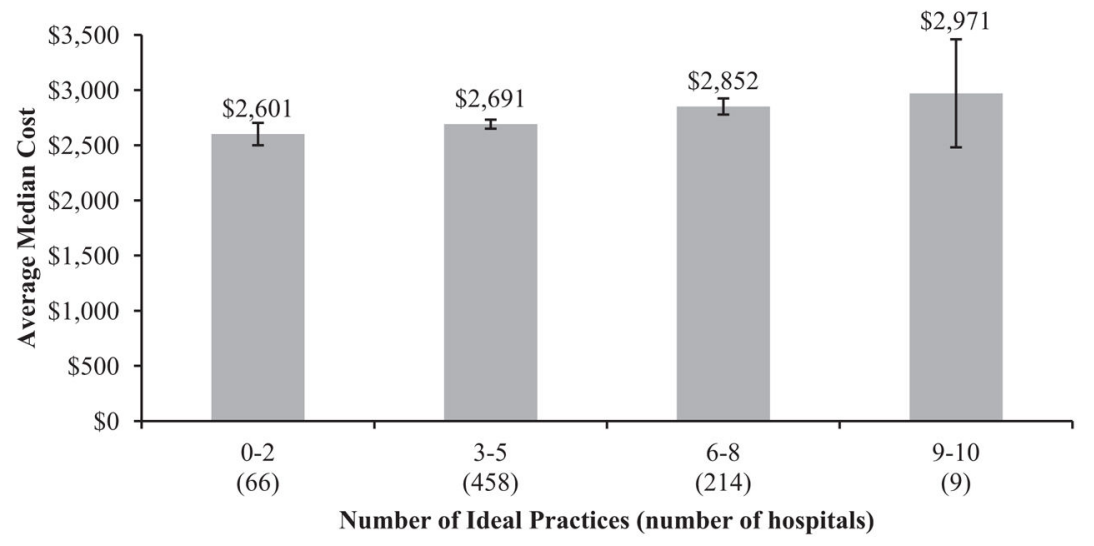

Figure 2.

Average median cost for uncomplicated vaginal births by number of ideal practices. *Ideal practices are indicators of the Ten Steps to Successful Breastfeeding. 


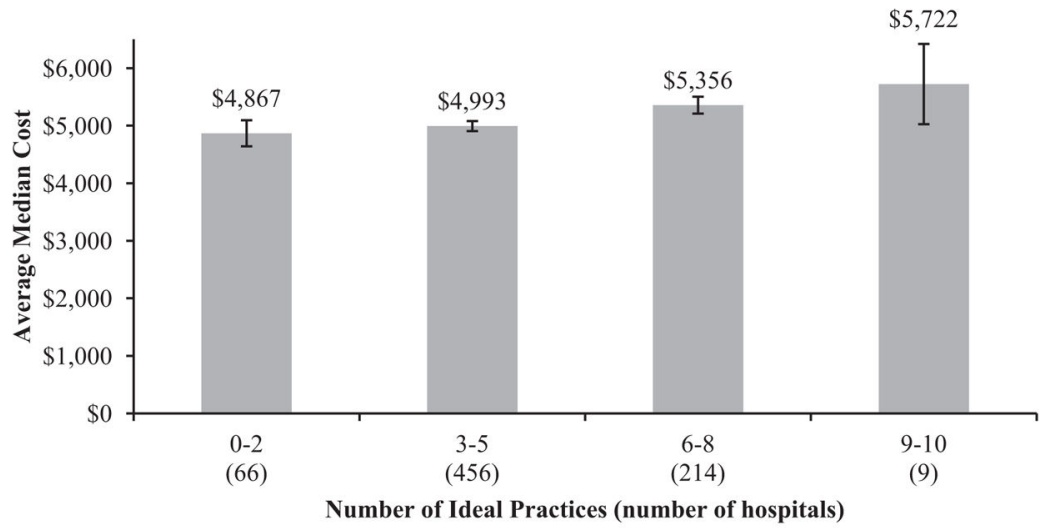

Figure 3.

Average median cost for uncomplicated cesarean section births by number of ideal practices. *Ideal practices are indicators of the Ten Steps to Successful Breastfeeding. 


\section{Table 1}

The Baby-Friendly steps for the U.S.

1 Have a written breastfeeding policy that is routinely communicated to all health care staff

2 Train all health care staff in the skills necessary to implement this policy

3 Inform all pregnant women about the benefits and management of breastfeeding

4 Help mothers initiate breastfeeding within an hour of birth

5 Show mothers how to breastfeed and maintain lactation, even if separated from their infant

6 Give newborn infants no food or drink other than breast milk, unless medically indicated

7 Practice rooming in-allowing mothers and infants to remain together 24 hours a day

8 Encourage breastfeeding on demand (encourage feeding on cue)

9 Give no pacifiers or artificial nipples to breastfeeding infants

10 Foster the establishment of breastfeeding support groups and refer mothers on discharge 
Table 2

Sample characteristics of facilities $(n=747)$.

\begin{tabular}{ll}
\hline Hospital type (\%) & 73 \\
Non-profit & 17 \\
Government & 10 \\
Private & 0 \\
Military & \\
Number of ideal maternity care practices * reported by facilities (\%) & 9 \\
$0-2$ & 61 \\
$3-5$ & 29 \\
$6-8$ & 1 \\
$9-10$ & 1488 \\
Mean number of annual births & 43 \\
Mean percent of Medicaid births & \\
* & \\
Ideal maternity care practices are indicators of the Ten Steps to Successful Breastfeeding.
\end{tabular}

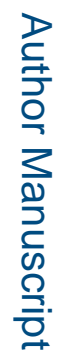

Birth. Author manuscript; available in PMC 2015 July 27. 

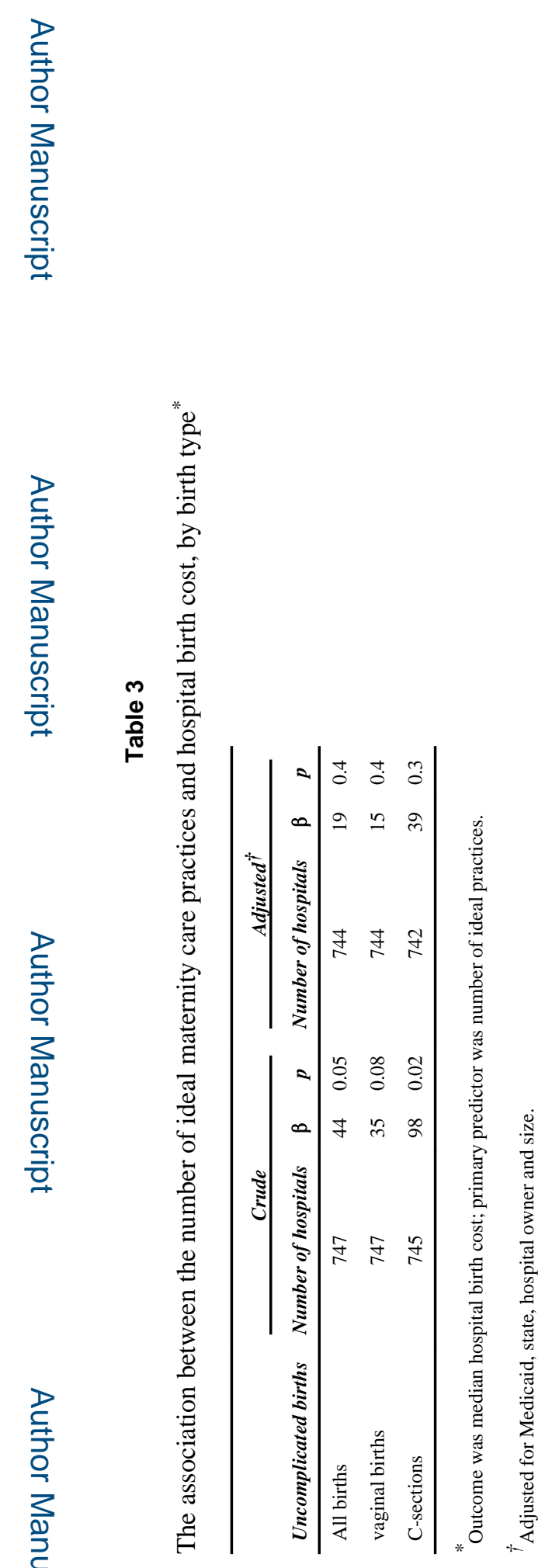

Birth. Author manuscript; available in PMC 2015 July 27. 